\title{
Clinical and demographic parameters predict the progression from mild cognitive impairment to dementia in elderly patients
}

\author{
Giovanni Zuliani ${ }^{1} \cdot$ Michele Polastri $^{2} \cdot$ Tommaso Romagnoli $^{2} \cdot$ Lisa Marabini $^{2} \cdot$ Davide Seripa $^{3} \cdot$ Carlo Cervellati $^{4}$. \\ Amedeo Zurlo $^{2} \cdot$ Angelina Passaro $^{2} \cdot$ Gloria Brombo $^{2}$
}

Received: 8 August 2020 / Accepted: 26 August 2020 / Published online: 12 September 2020

(c) The Author(s) 2020

\begin{abstract}
Objectives To evaluate the possibility of predicting the risk of progression from mild cognitive impairment (MCI) to dementia using a combination of clinical/demographic parameters.

Methods A total of $462 \mathrm{MCI}$ elderly patients (follow-up: 33 months). Variable measured included cognitive functions, age, gender, MCI type, education, comorbidities, clinical chemistry, and functional status.

Results Amnestic type (aMCI) represented 63\% of the sample, non-amnestic (naMCI) 37\%; 190 subjects progressed to dementia, 49\% among aMCI, and 28\% among naMCI. At Cox multivariate regression analysis, only MMSE (one point increase HR 0.84; 95\% CI 0.79-0.90), aMCI (HR 2.35; 95\% CI 1.39-3.98), and age (1 year increase HR 1.05; 95\% CI 1.01-1.10) were independently associated with progression to dementia. A score was created based on these dichotomized variables (score $0-3$ ): age ( $\geq$ or $<78$ years), MMSE score $(\geq$ or $<25 / 30)$ and aMCI type. The conversion rate progressed from $6 \%$ in subjects with score 0 (negative predictive value: 0.94 ), to $31 \%$ in individuals with score 1 , to $53 \%$ in subjects with score 2 , to $72 \%$ in individuals with score 3 (positive predictive value: 0.72 ). ROC curve analysis showed an area under the curve of 0.72 (95\% CI $0.66-0.75, p 0.0001)$.

Conclusions We have described a simple score, based on previously recognized predictors such as age, MMSE, and MCI type, which may be useful for an initial stratification of the risk of progression to dementia in patients affected by MCI. The score might help the clinicians to evaluate the need for more expansive/invasive examinations and for a closer follow-up in MCI patients.
\end{abstract}

Keywords Aging $\cdot$ Cox regression $\cdot$ Dementia $\cdot$ Follow-up $\cdot$ Mild cognitive impairment

Electronic supplementary material The online version of this article (https://doi.org/10.1007/s40520-020-01697-8) contains supplementary material, which is available to authorized users.

Giovanni Zuliani

zlngnn@unife.it

1 Department of Morphology, Surgery, and Medical Sciences, University of Ferrara, Azienda Ospedaliero-Universitaria S. Anna, 44100 Ferrara, Italy

2 Department of Medical Sciences, University of Ferrara, Ferrara, Italy

3 Research Laboratory, Complex Structure of Geriatrics, Department of Medical Sciences, Fondazione IRCCS Casa Sollievo Della Sofferenza, San Giovanni Rotondo, Italy

4 Department of Biomedical and Specialist Surgical Sciences, University of Ferrara, Ferrara, Italy

\section{Introduction}

Mild cognitive impairment (MCI) is an intermediate condition in the trajectory from normal cognition to dementia; compared to normal individuals, subjects with MCI usually have a higher rate of progression to dementia over a relatively short period [1]. The operational criteria for MCI diagnosis are the presence of a cognitive complain/impairment, an objective evidence of impairment in cognitive domains (e.g., memory, executive function/attention, language or visuospatial skills), essentially normal function in activities of daily living, and absence of dementia [1]. The reported prevalence of MCI over 60 years of age is approximately 7-25\% [2]; it increases with age and lower level of education, and seem to be more prevalent in men [2].

However, the prevalence of the preclinical phase of dementia may vary greatly, according to the diagnostic 
criteria and assessment procedures. In Italy, it has been estimated that up to $45 \%$ of population aged $65-84$ years had some kind of cognitive deficits without dementia [3].

Basically, a presentation with memory impairment is typical of the amnestic form of MCI (aMCI), whereas nonamnestic MCI (naMCI) is characterized by impairment in non-memory cognitive domains [1]. Currently, the majority of researchers employ four subtypes of MCI, depending on the number of affected domains: single-domain aMCI, multidomain aMCI, single-domain naMCI, and multidomain naMCI [4].

The issue of the progression to dementia is crucial, since the diagnosis of MCI implicates a prognosis that is "less favorable" compared to persons with normal cognition. Most of the studies report a rate of progression from 20 to $40 \%$, with an annual rate of $5-17 \%[1,2]$. However, while most patients who will develop dementia will exhibit symptoms compatible with MCI in the earlier stages of the disease [5], the reverse may not be true, since many individuals with MCI diagnosis may never progress to dementia [6]. Moreover, a small percentage of $\mathrm{MCI}$ individuals may revert to normal cognition, and even among these subjects, the risk of subsequent MCI or dementia is higher compared to healthy controls [7]. aMCI is hypothesized to progress mostly to Alzheimer's disease (AD), while naMCI usually progress to non-AD forms of dementia; both naMCI and aMCI have been associated with progression to vascular dementia (VD) [1]. Several risk factors have been associated with the possible progression from MCI to overt dementia in Literature including: older age, lower levels of formal education, memory loss or aMCI type, multiple domain MCI type, previous stroke, cerebrovascular disease, cardiovascular risk factors (e.g., hypertension, diabetes), presence of depression, poor health status, the degree of cognitive impairment, and perhaps female gender [1].

In the present study we evaluated, in a large sample of older individuals affected by MCI, the possibility of predicting the progression to dementia using a combination of easily accessible and inexpensive clinical/demographic parameters.

\section{Patients and methods}

\section{Subjects}

The initial sample included 620 elderly ( $\geq 65$ years) outpatients evaluated in the period 2006-2018 at the Memory Clinic of the Department of Internal Medicine, S. Anna University Hospital, Ferrara (Italy) or of the Casa Sollievo della Sofferenza, San Giovanni Rotondo (Italy) in which the diagnosis of MCI was made. After the initial examination and diagnosis, 158 subjects were lost during follow-up (we had no evidence from computerized health care system of a possible diagnosis of dementia), while 462 underwent a regular follow-up (median: 33 months; range 10-155). Of these, 190 converted to dementia, with a final rate of progression of about 30\% (190/620).

Subjects lost at follow-up did not differ from individuals included into the study as regards age, gender, education, MCI type, or prevalence of diabetes, hypertension, coronary heart disease (CHD), or stroke (data not shown).

MCI was defined as the presence of a documented deficit in memory or in another cognitive domain, without (single domain) or with (multiple domain) impairment in other cognitive domains, in an individual who didn't meet the clinical criteria for dementia [4].

The Mini-Mental State Examination (MMSE) [8] ranged from $22 / 30$ to $27 / 30$.

The clinical diagnosis of dementia during follow-up was made by the DSM-5 criteria [9].

LOAD diagnosis was based on the National Institute on Aging-Alzheimer's Association workgroups criteria [10], while the diagnosis of VD was made following the NINDS-AIREN criteria [11].

There were no evidence of acute illnesses at the time of clinical observation and blood sampling, as previously described $[12,13]$. No subject was taking NSAIDS, antibiotics, or steroids at the time of recruitment.

A cardiovascular risk score was calculated as follows: 1 point for active smoking, diabetes, hypertension and male gender; 2 points for previous diagnosis of coronary heart diseases or stroke (total score $0-8$ ).

General and neuropsychological examination including basic activities of daily living (BADLs) [14], instrumental activities of daily living (IADLs) [15], and 15 items geriatric depression scale (GDS) [16] was carried as previously described [17]. Personal data and medical history (e.g., hypertension, coronary heart disease-CHD, diabetes, chronic obstructive pulmonary disease-COPD) were collected by trained personnel as previously described [18]. Clinical chemistry analyses were routinely performed to exclude causes of secondary cognitive impairment. These analyses included serum B-12 vitamin and folate, liver, kidney and thyroid function tests, blood cell count, and arterial oxygen saturation.

All subjects underwent brain MRI or brain CT using a 64 volumetric scanner.

The study was approved by the Local Ethic Committee of "Casa Sollievo della Sofferenza", San Giovanni Rotondo (protocol n. 3877/DS) and Local Ethic Committee of "Azienda Arcispedale S. Anna", Ferrara (protocol n. 170579). Patients were informed about the project and research protocol, and a written consent was obtained. The research did not modify the routine clinical/diagnostic protocols implemented for the diagnosis of MCI 
nor conditioned any decision about the treatments of the enrolled individuals.

\section{Statistical analysis}

Continuous variables were expressed as mean (standard deviation-SD) or median (interquartile range-IQR) when necessary. Means were compared by ANOVA with Bonferroni post hoc test for multiple comparison; medians were compared by Mann-Whitney test. Correlations between continuous variables were tested by Pearson's correlation. Proportions were compared by the $\chi^{2}$ test. Hazard ratios (HR) were estimated by Cox proportional hazard regression analysis (univariate and multivariate). The assumption of proportionality of all variables introduced in the models was assessed through the analysis of Schoenfeld residuals. Analyses were performed by SPSS for Windows statistical package, version 13.0.

\section{Results}

Most of the sample was composed by aMCI patients (n.292-63\%), while the remaining subjects were affected by naMCI (n.170-37\%). The most frequent diagnosis was multidomain aMCI (54\%), followed by multidomain naMCI (25\%), single-domain naMCI (12\%), and single-domain aMCI (9\%). On the whole, 190 MCI progressed to dementia during the follow-up, about one in two among aMCI (49\%), and one in three among naMCI (28\%); in particular, the rate of progression was $29 \%$ for multiple naMCI, $34 \%$ for single naMCI, $48 \%$ for single aMCI, and $52 \%$ for multiple aMCI (Supplementary Fig. 1). Among the patients progressed to dementia, 34\% developed LOAD, 35\% "mixed" dementia (dementia with clinical-instrumental characteristics of both LOAD and VD), 29\% VD, and 2\% other forms of dementia.

In Table 1 are reported the principal characteristics of the sample according to clinical evolution. Compared to stable MCI, MCI converted to dementia were characterized by older age, higher prevalence of amnestic type, lower MMSE score, less depressive symptoms, and lower sub-cortical multiple lacunes. A non-significant trend toward an increase in the prevalence of diabetes, leukoaraiosis and brain atrophy was also observed.

In Table 2 are described the principal characteristics of the sample according to the clinical type of MCI. Compared with naMCI patients, aMCI were older, had less depressive symptoms, and lower hsCRP levels. The calculated cardiovascular score was generally low in the whole sample and did not differ between stable MCI patients or patients progressing to dementia, nor between aMCI and naMCI.

In Table 3 are reported the results of univariate Cox regression analysis for the conversion of MCI to dementia, based on the results reported in Table 1. Age (for 1 year increase-hazard ratio: 1.06 ; $95 \%$ confidence interval: 1.02-1.09), MMSE score (for 1 point increase-HR 0.88; 95\% CI 0.84-0.93), and type of MCI (amnestic vs nonamnestic-HR 2.38; 95\% CI 1.56-3.62) were significantly associated with the progression to dementia. On the contrary, the association between GDS score (three categories; $0-4,5-10,>10$ points) and progression was not significant.

In Table 4 are described the results of Cox multivariate regression model (stepwise forward-Wald) for progression of MCI to dementia. In order of entry into the model, MMSE score (for 1 point increase-HR 0.84; 95\% CI 0.79-0.90), amnestic type of MCI (HR 2.35; 95\% CI 1.39-3.98), and age (for 1 year increase-HR 1.05; 95\% CI 1.01-1.10) were associated with the progression to overt dementia, independent of sex and education; further adjustment for hs.CRP, multiple lacunar lesions and GDS score did not modify the results (data not shown).

In Fig. 1 are reported the survival curve obtained from Cox regression model for the progression to dementia in the MCI subjects grouped by the number (0-3) of significant predictors present in each individual: age ( $\geq$ or $<78$ years, median value), MMSE score ( $\geq$ or $<25 / 30$, median value), and aMCI type. Compared to subjects with 0 predictors, a graded and significant increase in the risk of progression to dementia was found in individuals bearing one factor (HR 12; 95\% CI 1.7-91), two factors (HR 28; 95\% CI 4.0-210) or three factors (HR 45; 95\% CI 6.5-328). Interestingly, the conversion rate progressed in a linear way from $6 \%$ in subjects with score 0 , to $31 \%$ in individuals with score $=1$, to $53 \%$ in subjects with score 2 , to $72 \%$ in individuals with score 3 . The ROC curve analysis for the score showed an area under the curve of 0.72 (95\% CI 0.66-0.75) with a standard error 0.030 ( $p$ 0.0001) (Supplementary Fig. 2).

\section{Discussion}

In this study, we searched for possible predictors of progression to dementia in a large sample of MCI elderly patients, focusing on easily accessible parameters. This is an important topic since the clinical diagnostic category "MCI" is heterogeneous. Indeed, it is known that while a part of individuals will progress to dementia, a large number of MCI will remain "stable" [5], and a part of them will even regress to normal cognitive functions [6].

In our study, about $30 \%$ of MCI patients evolved to dementia during the follow-up (median 33 months) with an observed maximum conversion rate between 1 and 3 years from baseline (almost 50\% of all cases).

At multivariate analysis, the diagnosis of aMCI was associated with a $135 \%$ increase in the probability of progression to dementia compared with naMCI. aMCI displayed a high 
Table 1 Principal characteristics of the sample according to follow-up evolution

\begin{tabular}{|c|c|c|c|}
\hline & MCI-MCI ( $n$ 272) & MCI-dementia ( $n$ 190) & $p$ \\
\hline \multicolumn{4}{|l|}{ Demographics } \\
\hline Age (years) & $76.6 \pm 6.3$ & $78.3 \pm 4.7$ & 0.002 \\
\hline Female gender $(\%)$ & 54 & 59 & 0.33 \\
\hline Education (years) & $6.1 \pm 3.7$ & $6.0 \pm 3.4$ & 0.75 \\
\hline Active smoking (\%) & 7.8 & 8.0 & 0.96 \\
\hline \multicolumn{4}{|l|}{ Cognitive and functional status } \\
\hline MMSE (/30) & $25.3 \pm 2.7$ & $24.0 \pm 2.8$ & 0.001 \\
\hline GDS (/15) & $5(3-7)$ & $3(2-6)$ & 0.007 \\
\hline BADLs (/6) & $5.4 \pm 1.0$ & $5.1 \pm 1.2$ & 0.80 \\
\hline IADLs (/8) & $6.4 \pm 2.1$ & $5.9 \pm 2.5$ & 0.18 \\
\hline Amnestic type MCI (within group) (\%) & 55 & 74.5 & 0.001 \\
\hline \multicolumn{4}{|l|}{ Clinical chemistry parameters } \\
\hline Haemoglobin $(\mathrm{g} / \mathrm{dl})$ & $13.1 \pm 1.4$ & $13.1 \pm 1.4$ & 0.98 \\
\hline Creatinine (mg/dl) & $0.94 \pm 0.27$ & $0.95 \pm 0.29$ & 0.86 \\
\hline Uric acid (mg/dl) & $4.9 \pm 1.6$ & $5.1 \pm 1.2$ & 0.64 \\
\hline Albumin (g/dl) & $4.0 \pm 0.33$ & $4.0 \pm 0.35$ & 0.59 \\
\hline Total cholesterol (mg/dl) & $205 \pm 40$ & $207 \pm 41$ & 0.68 \\
\hline Tryglicerides (mg/dl) & $107 \pm 38$ & $119 \pm 67$ & 0.0 \\
\hline HDL-C (mg/dl) & $60 \pm 16$ & $64 \pm 38$ & 0.23 \\
\hline Hs-CRP (mg/dl) & $0.18(0.12-0.25)$ & $0.16(0.10-0.27)$ & 0.46 \\
\hline Glycemia (mg/dl) & $98 \pm 21$ & $103 \pm 41$ & 0.25 \\
\hline HbA1c (\%) & $5.9 \pm 0.8$ & $6.1 \pm 1.3$ & 0.15 \\
\hline Cardiovascular Score & $1.68 \pm 1.1$ & $1.64 \pm 1.1$ & 0.75 \\
\hline Homocysteine $(\mu \mathrm{mol} / \mathrm{l})$ & $15(12-18)$ & $16(14-20)$ & 0.10 \\
\hline Vitamin B12 (pg/ml) & $302(247-387)$ & $309(263-283)$ & 0.92 \\
\hline Folic acid (ng/ml) & $6(5-7)$ & $5.9(5.1-7)$ & 0.78 \\
\hline \multicolumn{4}{|l|}{ Comorbidities } \\
\hline Hypertension (\%) & 56 & 58 & 0.97 \\
\hline Coronary heart disease $(\%)$ & 14.2 & 13.5 & 0.86 \\
\hline Diabetes $(\%)$ & 14.8 & 20.1 & 0.15 \\
\hline Stroke $(\%)$ & 4.1 & 5.3 & 0.64 \\
\hline COPD $(\%)$ & 6.1 & 6.1 & 0.98 \\
\hline \multicolumn{4}{|l|}{ MRI parameters } \\
\hline Cortical lesions (\%) & 13.6 & 12.8 & 0.67 \\
\hline Single lacunar lesion (\%) & 7 & 8 & 0.72 \\
\hline Multiple lacunar lesions (\%) & 42 & 31 & 0.04 \\
\hline Leukoaraiosis (\%) & 33 & 42 & 0.14 \\
\hline Atrophy (\%) & 49 & 57 & 0.21 \\
\hline
\end{tabular}

Continuous variables are expressed as mean \pm SEM or median (interquartile range). Categorical variables are expressed as percentage within group

MMSE mini mental state examination, GDS global deterioration scale, IADL instrumental activities of daily living, $B A D L$ basic activity of daily living, $H D L-C$ high density lipoprotein cholesterol, $H s-C R P$ high-sensitivity $\mathrm{C}$-reactive protein, $\mathrm{HbAlc}$ hemoglobin $\mathrm{A} 1 \mathrm{c}$ rate of progression (49\%), and predominantly progressed to LOAD (33\%) or "mixed" dementia (44\%). Conversely, naMCI showed a lower rate of conversion (28\%), and mainly evolved to VD $(25 \%)$ or "mixed dementia" (43\%). These findings are in good agreement with previous observations $[18,19]$. However, as previously noted by Fischer et al. [20], the subtypes of MCI were not very useful in defining with precision the evolution to different types of dementia; indeed, both aMCI and naMCI progressed to LOAD, while the evolution to VD was not restricted to naMCI.

Age was another independent predictor of MCI conversion, with an increase of the risk of $5 \%$ per year at multivariate analysis. In particular, individuals with age $\geq 78$ years (median age in our sample) had an $87 \%$ risk increase 
Table 2 Principal characteristics of the sample according to MCI type

\begin{tabular}{|c|c|c|c|}
\hline & Amnestic MCI ( $n$ 292) & Non-amnestic MCI ( $n$ 170) & $p$ \\
\hline \multicolumn{4}{|l|}{ Demographics } \\
\hline Age (years) & $77.7 \pm 5.6$ & $76.2 \pm 5.8$ & 0.01 \\
\hline Female gender $(\%)$ & 57 & 61 & 0.24 \\
\hline Education (years) & $6.0 \pm 3.4$ & $6.1 \pm 3.6$ & 0.77 \\
\hline Active smoking (\%) & 8.5 & 8.1 & 0.96 \\
\hline \multicolumn{4}{|l|}{ Cognitive and functional status } \\
\hline MMSE (/30) & $24.6 \pm 2.7$ & $24.2 \pm 3.3$ & 0.18 \\
\hline GDS (/15) & $4(3-6)$ & $5(3-6)$ & 0.05 \\
\hline BADLs (/6) & $5.4 \pm 1.0$ & $5.2 \pm 1.3$ & 0.85 \\
\hline IADLs (/8) & $5.9 \pm 2.4$ & $5.6 \pm 2.7$ & 0.40 \\
\hline \multicolumn{4}{|l|}{ Clinical chemistry parameters } \\
\hline Haemoglobin $(\mathrm{g} / \mathrm{dl})$ & $13.1 \pm 1.4$ & $13.1 \pm 1.4$ & 0.98 \\
\hline Creatinine (mg/dl) & $1.01 \pm 0.27$ & $0.94 \pm 0.29$ & 0.74 \\
\hline Uric acid (mg/dl) & $5.0 \pm 1.8$ & $5.3 \pm 1.4$ & 0.41 \\
\hline Albumin (g/dl) & $4.0 \pm 0.37$ & $4.0 \pm 0.31$ & 0.61 \\
\hline Total cholesterol (mg/dl) & $207 \pm 45$ & $207 \pm 40$ & 0.97 \\
\hline Tryglicerides (mg/dl) & $117 \pm 54$ & $116 \pm 57$ & 0.82 \\
\hline HDL-C (mg/dl) & $61 \pm 15$ & $58 \pm 29$ & 0.28 \\
\hline Hs-CRP (mg/dl) & $0.15(0.10-0.25)$ & $0.20(0.14-0.38)$ & 0.02 \\
\hline Glycemia (mg/dl) & $98 \pm 18$ & $103 \pm 40$ & 0.11 \\
\hline HbA1c $(\%)$ & $5.9 \pm 0.8$ & $6.1 \pm 1.3$ & 0.15 \\
\hline Cardiovascular Score & $1.66 \pm 1.2$ & $1.70 \pm 1.1$ & 0.68 \\
\hline Homocysteine $(\mu \mathrm{mol} / \mathrm{l})$ & $15(12-19)$ & $15(13-18)$ & 0.40 \\
\hline Vitamin B12 (pg/ml) & $308(252-391)$ & $322(278-390)$ & 0.18 \\
\hline Folic acid (ng/ml) & $5.9(4.9-7.1)$ & $6.1(5.1-7.3)$ & 0.46 \\
\hline \multicolumn{4}{|l|}{ Comorbidities } \\
\hline Hypertension (\%) & 56 & 58 & 0.97 \\
\hline Coronary heart disease $(\%)$ & 13.7 & 14.2 & 0.81 \\
\hline Diabetes (\%) & 17.6 & 14.5 & 0.37 \\
\hline Stroke $(\%)$ & 4.3 & 4.9 & 0.78 \\
\hline COPD $(\%)$ & 6.6 & 6.2 & 0.83 \\
\hline \multicolumn{4}{|l|}{ MRI parameters } \\
\hline Cortical lesions (\%) & 13.5 & 12.0 & 0.64 \\
\hline Single lacunar lesion (\%) & 8 & 7 & 0.77 \\
\hline Multiple lacunar lesions (\%) & 33 & 37 & 0.68 \\
\hline Leukoaraiosis $(\%)$ & 36 & 40 & 0.45 \\
\hline Atrophy (\%) & 56 & 52 & 0.43 \\
\hline
\end{tabular}

Continuous variables are expressed as mean \pm SEM or median (interquartile range). Categorical variables are expressed as percentage within group

MMSE mini mental state examination, GDS global deterioration scale, IADL instrumental activities of daily living, $B A D L$ basic activity of daily living, $H D L-C$ high density lipoprotein cholesterol, $H s-C R P$ high-sensitivity C-reactive protein, $H b A 1 c$ hemoglobin A1c compared with younger subjects. This finding was expected since the incidence of dementia has been associated with age in MCI individuals [18, 21-23].

The degree of the cognitive impairment at baseline was also strongly associated with the progression to dementia; a reduction of 1 point in MMSE score was independently associated with a $16 \%$ increase in the risk of progression. Individuals with MMSE score $<25 / 30$ (median value in our sample) displayed an increase of $151 \%$ in the risk of developing dementia compared with subjects with MMSE $\geq 25 / 30$. Although our results are in agreement with previous studies [18, 24-26], it has to be noted that a metanalysis by Arevalo-Rodriguez et al. [27] found that the accuracy of baseline MMSE score for conversion to dementia generally was low, with a sensitivity of $23-76 \%$ and specificity of $40-94 \%$. 
Table 3 Univariate Cox regression models for conversion of MCI to dementia

\begin{tabular}{lllll}
\hline Variables & $B(\mathrm{SE})$ & $\mathrm{HR}$ & $95 \% \mathrm{CI}$ & $p$ \\
\hline Age (1 year) & $0.057(0.015)$ & 1.06 & $1.02-1.09$ & 0.0001 \\
MMSE (1 point) & $-0.12(0.027)$ & 0.88 & $0.84-0.93$ & 0.0001 \\
aMCI type & $0.87(0.21)$ & 2.38 & $1.56-3.62$ & 0.0001 \\
GDS score & & & & \\
$0-4$ & - & 1 & - & \\
$5-10$ & $-0.28(0.20)$ & 0.75 & $0.50-1.11$ & \\
$>10$ & $-0.16(0.30$ & 0.85 & $0.47-1.54$ & 0.35 \\
\hline
\end{tabular}

MMSE mini mental state examination, GDS global deterioration scale, $S E$ standard error, $H R$ hazard ratio, $C I$ confidential index

Based on these findings, we created a simple individual prognostic score based on the presence of three parameters including aMCI type, age $\geq 78$ years, and MMSE $<25 / 30$. The score stratified our MCI population in four group with increasing risk of future evolution to dementia:

- MCI patients with score 0 ( $13 \%$ of sample) had a very low probability of evolution to dementia, with a very high negative predictive value (0.94). Of consequence, they should be reassured and followed in a less pressing way compared with individuals with higher scores.

- MCI patients with score 1 (28\% of sample) had a low probability of evolution to dementia, with a high negative predictive value (0.69).

- MCI patients with score 2 (42\% of sample) had an intermediate probability of progression, with a high positive predictive value (0.53).

- MCI patients with score 3 (17\% of sample) had a high probability of progression, with a high positive predictive value (0.72). These subjects should be deeply investigated to exclude a possible underlying diagnosis of initial dementia. Moreover, these patients might gain much more benefit from specific therapies, although at present no drugs are specifically approved for MCI treatment.

We also calculated a cardiovascular risk score based on well-known risk factors and vascular comorbidities (CHD and stroke). Although the cardiovascular risk has been associated with both MCI [28], and progression from MCI to dementia [29] in our sample the cardiovascular risk score was not predictive of the progression to dementia.

Finally, we must acknowledge an important limitation of the study. In evaluating the risk of progression to dementia we did not include some comorbidities among possible confounders, such as heart failure and atrial fibrillation. Indeed, a continuum among hypertension, coronary artery disease, atrial fibrillation, and chronic heart failure with the development of cognitive impairment and progression to dementia has been hypothesized [30]. Moreover, it has been demonstrated not only that atrial fibrillation predicts evolution to dementia in elderly subjects with MCI, but also that ventricular rate response seems to play a key role in dementia incidence among patients with atrial fibrillation [31].

We would also underline some strength of the study, in particular the large size of the MCI sample (over 450 individuals) and the length the follow-up (median length 33 months).

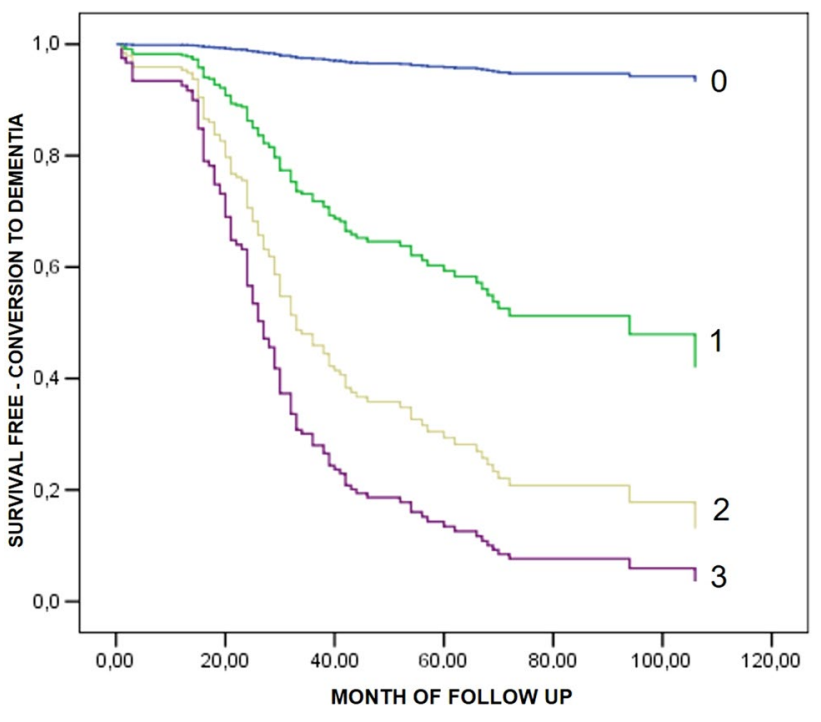

Fig. 1 Prognosis of dementia by number of predictors (0-3) including age $>78$ years, MMSE $<25 / 30$, and amnestic type of MCI
Table 4 Multivariate Cox regression model (stepwise forward Wald) for conversion of MCI to dementia

\begin{tabular}{lrlllll}
\hline Variables & \multicolumn{1}{l}{$B(\mathrm{SE})$} & $\mathrm{HR}$ & $95 \%$ CI & $R$ squared & Total $R$ squared & $p$ \\
\hline 1. MMSE & $-0.164(0.034)$ & 0.84 & $0.79-0.90$ & 24.73 & 24.73 & 0.0001 \\
2. aMCI type & $0.827(0.268)$ & 2.35 & $1.39-3.98$ & 13.20 & 37.93 & 0.0001 \\
3. Age & $0.057(0.021)$ & 1.05 & $1.01-1.10$ & 7.50 & 45.44 & 0.0001 \\
\hline
\end{tabular}

Adjusting covariates: age, sex, MCI type, MMSE (continuous variable), education

$M M S E$ mini mental state examination, $S E$ standard error, $H R$ hazard ratio, $C I$ confidential index 


\section{Conclusion}

In conclusion, we have described a simple score, based on previously recognized predictors such as age, MMSE score, and type of MCI, which may be useful for an initial stratification of the risk of progression to dementia in patients affected by MCI. The score might help the clinician to evaluate the need for more expansive/invasive examinations and for a closer follow-up in MCI patients.

Funding Open access funding provided by Università degli Studi di Ferrara within the CRUI-CARE Agreement.

\section{Compliance with ethical standards}

Conflict of interest The authors declare that there is no conflict of interest.

Human and animal rights The study was approved by the Local Ethic Committee of "Casa Sollievo della Sofferenza", San Giovanni Rotondo (protocol n. 3877/DS) and Local Ethic Committee of "Azienda Arcispedale S. Anna”, Ferrara (protocol n. 170579). Study procedures were performed in accordance with the Declaration of Helsinki ethical principles for medical research involving human subjects.

Informed consent All participants provided written informed consent.

Open Access This article is licensed under a Creative Commons Attribution 4.0 International License, which permits use, sharing, adaptation, distribution and reproduction in any medium or format, as long as you give appropriate credit to the original author(s) and the source, provide a link to the Creative Commons licence, and indicate if changes were made. The images or other third party material in this article are included in the article's Creative Commons licence, unless indicated otherwise in a credit line to the material. If material is not included in the article's Creative Commons licence and your intended use is not permitted by statutory regulation or exceeds the permitted use, you will need to obtain permission directly from the copyright holder. To view a copy of this licence, visit http://creativecommons.org/licenses/by/4.0/.

\section{References}

1. Roberts R, Knopman DS (2013) Classification and epidemiology of MCI. Clin Geriatr Med 29:753-772. https://doi. org/10.1016/j.cger.2013.07.003

2. Jongsiriyanyong S, Limpawattana P (2018) Mild cognitive impairment in clinical practice: a review article. Am J Alzheimer's Dis Other Demen 33:500-507. https://doi. org/10.1177/1533317518791401

3. Scafato E, Gandin C, Galluzzo L et al (2010) Prevalence of aging-associated cognitive decline in an Italian elderly population: results from cross-sectional phase of Italian Project on Epidemiology of Alzheimer's disease (IPREA). Aging Clin Exp Res. https://doi.org/10.1007/BF03337739

4. Petersen RC, Negash S (2008) Mild cognitive impairment: an overview. CNS Spectr 13:45-53. https://doi.org/10.1017/S1092 852900016151
5. Forlenza OV, Chiu E (2008) Mild cognitive impairment: a concept ready to move on? Curr Opin Psychiatry 21:529-532. https ://doi.org/10.1097/YCO.0b013e328316c2ab

6. Forlenza OV, Diniz BS, Gattaz WF (2010) Diagnosis and biomarkers of predementia in Alzheimer's disease. BMC Med 8:89. https://doi.org/10.1186/1741-7015-8-89

7. Koepsell TD, Monsell SE (2012) Reversion from mild cognitive impairment to normal or near-normal cognition: risk factors and prognosis. Neurology 79:1591-1598. https://doi.org/10.1212/ WNL.0b013e31826e26b7

8. Folstein MF, Folstein SE, McHugh PR (1975) Mini-mental state. J Psychiatr Res 12:189-198. https://doi.org/10.1016/00223956(75)90026-6

9. American Psychiatric Association (2013) Diagnostic and statistical manual of mental disorders (5th ed). https://doi. org/10.1176/appi.books.9780890425596

10. McKhann GM, Knopman DS, Chertkow H et al (2011) The diagnosis of dementia due to Alzheimer's disease: recommendations from the National Institute on Aging-Alzheimer's Association workgroups on diagnostic guidelines for Alzheimer's disease. Alzheimers Dement 7:263-269. https://doi.org/10.1016/j. jalz.2011.03.005

11. Román GC, Tatemichi TK, Erkinjuntti T et al (1993) Vascular dementia: diagnostic criteria for research studies. Report of the NINDS-AIREN International Workshop. Neurology 43:250-260. https://doi.org/10.1212/WNL.43.2.250

12. Cervellati C, Romani A, Bergamini CM et al (2015) PON-1 and ferroxidase activities in older patients with mild cognitive impairment, late onset Alzheimer's disease or vascular dementia. Clin Chem Lab Med 53:1049-1056. https://doi.org/10.1515/ cclm-2014-0803

13. Castellazzi M, Trentini A, Romani A et al (2016) Decreased arylesterase activity of paraoxonase-1 (PON-1) might be a common denominator of neuroinflammatory and neurodegenerative diseases. Int J Biochem Cell Biol 81:356-363. https://doi. org/10.1016/j.biocel.2016.06.008

14. Katz S (1963) Studies of illness in the aged. JAMA 185:914. https ://doi.org/10.1001/jama.1963.03060120024016

15. Lawton MP, Brody EM (1969) Assessment of older people: selfmaintaining and instrumental activities of daily living. Gerontologist 9:179-186. https://doi.org/10.1093/geront/9.3_Part_1.179

16. Yesavage JA, Sheikh JI (1986) 9/Geriatric depression scale (GDS). Clin Gerontol 5:165-173. https://doi.org/10.1300/J018v 05n01_09

17. Cervellati C, Trentini A, Rosta V et al (2020) Serum betasecretase 1 (BACE1) activity as candidate biomarker for lateonset Alzheimer's disease. GeroScience 42:159-167. https://doi. org/10.1007/s11357-019-00127-6

18. Ravaglia G, Forti P, Montesi F et al (2008) Mild cognitive impairment: epidemiology and dementia risk in an elderly Italian population. J Am Geriatr Soc 56:51-58. https://doi.org/10.111 1/j.1532-5415.2007.01503.x

19. Palmer K, Bäckman L, Winblad B et al (2008) Mild cognitive impairment in the general population: occurrence and progression to Alzheimer disease. Am J Geriatr Psychiatry 16:603-611. https ://doi.org/10.1097/JGP.0b013e3181753a64

20. Hussain $\mathrm{H}$ (2007) Conversion from subtypes of mild cognitive impairment to Alzheimer dementia. Neurology 69:409. https:// doi.org/10.1212/01.wnl.0000278072.42014.6d

21. Artero S, Ancelin M-L, Portet F et al (2008) Risk profiles for mild cognitive impairment and progression to dementia are gender specific. J Neurol Neurosurg Psychiatry 79:979-984. https://doi. org/10.1136/jnnp.2007.136903

22. Manly JJ, Tang M-X, Schupf N et al (2008) Frequency and course of mild cognitive impairment in a multiethnic community. Ann Neurol 63:494-506. https://doi.org/10.1002/ana.21326 
23. Plassman BL, Langa KM, Fisher GG et al (2008) Prevalence of cognitive impairment without dementia in the United States. Ann Intern Med 148:427-434. https://doi.org/10.7326/0003-4819-1486-200803180-00005

24. Han JW, Kim TH, Lee SB et al (2012) Predictive validity and diagnostic stability of mild cognitive impairment subtypes. Alzheimer's Dement 8:553-559. https://doi.org/10.1016/j. jalz.2011.08.007

25. Lopez OL, Jagust WJ, DeKosky ST et al (2003) Prevalence and classification of mild cognitive impairment in the cardiovascular health study cognition study. Arch Neurol 60:1385. https://doi. org/10.1001/archneur.60.10.1385

26. Peters ME, Rosenberg PB, Steinberg M et al (2013) Neuropsychiatric symptoms as risk factors for progression from CIND to dementia: the cache county study. Am J Geriatr Psychiatry 21:1116-1124. https://doi.org/10.1016/j.jagp.2013.01.049

27. Arevalo-Rodriguez I, Smailagic N, Roquéi Figuls M et al (2015) Mini-Mental State Examination (MMSE) for the detection of Alzheimer's disease and other dementias in people with mild cognitive impairment (MCI). Cochrane Database Syst Rev 2015:CD010783. https://doi.org/10.1002/14651858.CD010783. pub2
28. Wang Y, Song M, Yu L et al (2015) Mild cognitive impairment: vascular risk factors in community elderly in four cities of Hebei Province. China PLoS One 10:e0124566. https://doi.org/10.1371/ journal.pone.0124566

29. Ettorre E, Cerra E, Marigliano B et al (2012) Role of cardiovascular risk factors (CRF) in the patients with mild cognitive impairment (MCI). Arch Gerontol Geriatr 54:330-332. https:// doi.org/10.1016/j.archger.2011.04.025

30. Abete P, Della-Morte D, Gargiulo G et al (2014) Cognitive impairment and cardiovascular diseases in the elderly. A heart-brain continuum hypothesis. Ageing Res Rev 18:41-52. https://doi. org/10.1016/j.arr.2014.07.003

31. Cacciatore F, Testa G, Langellotto A et al (2012) Role of ventricular rate response on dementia in cognitively impaired elderly subjects with atrial fibrillation: a 10-year study. Dement Geriatr Cogn Disord 34:143-148. https://doi.org/10.1159/000342195

Publisher's Note Springer Nature remains neutral with regard to jurisdictional claims in published maps and institutional affiliations. 